03

\title{
Применение модифицированного метода дискретных источников и метода диаграммных уравнений к решению задачи дифракции волн на теле с шероховатой границей
}

\author{
(C) А.Г. Кюркчан ${ }^{1,2,3}$, С.А. Маненков ${ }^{19}$ \\ ${ }^{1}$ Московский технический университет связи и инорорматики, \\ 111024 Москва, Россия \\ ${ }^{2}$ Фрязинский филиал Института радиотехники и электроники им. В.А. Котельникова РАН, \\ 141190 Фрязино, Московская обл., Россия \\ ${ }^{3}$ ФГУП Центральный научно-исследовательский институт связи, \\ 111141 Москва, Россия \\ T e-mail:mail44471@mail.ru
}

Поступила в редакцию 04.04.2021 г.

В окончательной редакции 10.05.2021 г.

Принята к публикации 14.05.2021 г.

Рассмотрена двумерная задача дифракции на цилиндрическом теле с шероховатой границей. В работе исследованы два аспекта задачи дифракции на теле с неровной границей. Во-первых, изучена задача дифракции на телах со случайными возмущениями границы. В качестве примера рассмотрена дифракция на шероховатом круговом цилиндре. Проведено сравнение результатов расчета усредненной диаграммы рассеяния, полученной при помощи модифицированного метода дискретных источников, с результатами, полученными с использованием метода малых возмущений. Второй целью работы является выяснение степени влияния малых возмущений границы рассеивателя на геометрию множества особенностей аналитического продолжения дифракционного поля. В качестве примера рассмотрена задача дифракции на цилиндрическом теле с сечением в виде шероховатого многолистника, заданного в полярных и эллиптических координатах.

Ключевые слова: дифракция волн на телах с шероховатой границей, метод дискретных источников, аналитическое продолжение волновых полей.

DOI: $10.21883 /$ OS.2021.09.51343.2113-21

\section{Введение}

В литературе имеется большое количество публикаций, посвященных дифракции волн на телах и поверхностях с неровной границей (в том числе описываемой случайной функцией координат) [1-7]. В настоящей работе исследуется двумерная задача дифракции волн на бесконечном цилиндрическом теле с шероховатой границей. Как известно, существует множество методов решения такой задачи. Большая группа методов основана на использовании того или иного малого параметpa $[1-5,7]$. Наличие такого параметра накладывает достаточно жесткие условия на степень „шероховатости“ границы рассеивателя. В работе на основе модифицированного метода дискретных источников (ММДИ) [810] предложен эффективный строгий подход к решению задачи дифракции на статистически неровном теле. При этом неровности границы моделировались при помощи синусоидальной функции, имеющей случайную амплитуду и независимую от нее случайную фазу. В качестве примера была рассмотрена дифракция плоской волны на шероховатом круговом цилиндре. В предположении малости амплитуды неровностей по сравнению с длиной волны было проведено сравнение результатов расчета усредненной диаграммы, полученной при помо- щи ММДИ и при помощи метода малых возмущений (MМB) [1]. В последнем случае для вывода расчетных соотношений использовалась методика, основанная на методе диаграммных уравнений (МДУ) [9,11]. Была рассмотрена также дифракция на статистически неровных цилиндрических телах с сечением в виде различных многолистников. Соответствующая задача решалась при помощи ММДИ. Отметим, что, с одной стороны, ММДИ имеет преимущество, например, перед методом токовых интегральных уравнений, так как не требует вычисления интегралов при нахождении матричных элементов соответствующей алгебраической системы. Этот факт очень важен при усреднении диаграммы рассеяния в случае статистически неровной границы тела. С другой стороны, метод позволяет решать задачу дифракции на теле с аналитической границей с высокой точностью.

Ранее в работе [12] была сделана попытка дать объяснение феномена распознавания зеркальных объектов, т. е. рассеивателей, имеющих идеально гладкую границу. Если говорить коротко, то мы распознаем зеркальные объекты за счет изображения в них находящихся рядом предметов. Очевидно, что идеально зеркальных объектов в природе не существует. Все они в той или иной степени „шероховатые“. Представляет интерес вопрос о том, как влияет форма сечения „невозмущенного 
тела (при условии малости неровностей границы по сравнению с длиной волны) на то, чтобы шероховатый рассеиватель воспринимался как идеально отражающий или зеркальный. Как известно, рассеянное каким-либо объектом поле порождается источниками, расположенными на поверхности и (или) внутри рассеивателя. Если объект относится к категории „зеркальных“, то источники рассеянного им поля расположены внутри рассеивателя [9]. В настоящей работе сделана попытка определить, при каком условии на форму сечения объект может быть отнесен к зеркальным. При этом в качестве критерия была выбрана зависимость геометрии множества особенностей аналитического продолжения дифракционного поля внутрь области, занимаемой телом, и диаграммы рассеяния от формы сечения рассеивателя.

В работах $[13,14]$ ММДИ применялся к задаче дифракции на различных многолистниках вращения, заданных в сферических и сфероидальных координатах. В этих работах был предложен численный алгоритм построения вспомогательного контура осевого сечения тела, являющегося носителем дискретных источников. Однако этот подход пригоден лишь для случая наличия симметрии контура тела относительно обеих координатных осей. Если же тело не симметрично, то указанная методика не применима. В настоящей работе алгоритм построения вспомогательного контура модифицирован таким образом, чтобы он был пригоден для решения задачи дифракции на телах с неровной границей, описываемой отрезком ряда Фурье, который содержит гармоники с произвольными амплитудами и фазами. Это позволяет применить ММДИ к широкому классу тел с шероховатой границей. Необходимо лишь, чтобы функция, задающая уравнение границы тела, была аналитической функцией координат.

\section{Постановка задачи и вывод интегрального уравнения}

Рассмотрим постановку задачи. Требуется найти рассеянное волновое поле $U^{1}$ при дифракции плоской волны

$$
U^{0}=\exp \left(-i k r \cos \left(\varphi-\varphi_{0}\right)\right)
$$

где $k-$ волновое число, а $\varphi_{0}-$ угол падения волны, на бесконечном цилиндрическом теле, на поверхности которого задано краевое условие

$$
\left.\left(\frac{\partial U}{\partial n}-Z U\right)\right|_{S}=0
$$

где $U=U^{0}+U^{1}, S-$ контур сечения тела, $Z-$ импеданс на поверхности тела. Вторичное (рассеянное) поле удовлетворяет условию излучения на бесконечности:

$$
\lim _{r \rightarrow \infty} \sqrt{r}\left(\frac{\partial U^{1}}{\partial r}+i k U^{1}\right)=0 .
$$

При этом предполагается, что контур $S$ описывается уравнением

$$
\mathbf{r}_{S}=\mathbf{r}_{S}^{0}+\Delta \mathbf{r}\left(\mathbf{r}_{S}^{0}\right)
$$

где $\mathbf{r}_{S}^{0}$ - радиус-вектор точки на „невозмущенном“ контуре $S_{0}, \Delta \mathbf{r}\left(\mathbf{r}_{S}^{0}\right)$ - малая добавка (возмущения границы тела). Заметим, что приведенный ниже алгоритм на основе ММДИ не накладывает ограничения на длину волны падающего поля.

Будем решать поставленную задачу с помощью ММДИ. Для этого представим волновое поле вне тела в виде

$$
U^{1}(\mathbf{r})=\int_{\Sigma} G\left(\mathbf{r}, \mathbf{r}^{\prime}\right) j\left(\mathbf{r}^{\prime}\right) d s^{\prime} .
$$

Здесь $j\left(\mathbf{r}^{\prime}\right)$ - неизвестный ток на вспомогательном контуре $\Sigma$, который расположен внутри контура поперечного сечения тела $S$. Выбор контура $\Sigma$ будет рассмотрен ниже. В формуле (5) $G\left(\mathbf{r}, \mathbf{r}^{\prime}\right)$ - функция Грина, которая имеет вид

$$
G\left(\mathbf{r}, \mathbf{r}^{\prime}\right)=-\frac{i}{4} H_{0}^{(2)}\left(k \sqrt{\left(x-x^{\prime}\right)^{2}+\left(y-y^{\prime}\right)^{2}}\right) .
$$

Подставив формулу (5) в граничное условие (2), получим интегральное уравнение первого рода относительно функции $j\left(\mathbf{r}^{\prime}\right)$

$$
\begin{gathered}
\int_{\Sigma}\left(\frac{\partial G\left(\mathbf{r}, \mathbf{r}^{\prime}\right)}{\partial n}-Z G\left(\mathbf{r}, \mathbf{r}^{\prime}\right)\right) j\left(\mathbf{r}^{\prime}\right) d s^{\prime}= \\
=-\left(\frac{\partial U^{0}(\mathbf{r})}{\partial n}-Z U^{0}(\mathbf{r})\right), \quad \mathbf{r} \in S .
\end{gathered}
$$

Для алгебраизации задачи применим метод коллокации $[8,9,10,13,14]$. Пусть контур сечения тела задан в некоторой ортогональной системе координат $(\alpha, \beta)$, где $\beta \in[0,2 \pi]$. В соответствии с методом коллокации мы разбиваем интервал изменения переменной $\beta$ точками деления $\beta_{n} \equiv \frac{2 \pi}{N}(n-1 / 2)$, где $n=1,2, \ldots, N$. Далее заменяем интеграл в уравнении (7) суммой Римана и приравниваем левую и правую части в полученном уравнении в точках коллокации, которые выбираем при $\beta=\beta_{n}$. Тем самым мы переходим от интегрального представления поля к дискретным источникам, расположенным на вспомогательном контуре внутри сечения тела.

\section{Выбор вспомогательного контура в подходящих ортогональных координатах}

Пусть контур сечения тела $S$ задан в ортогональных координатах $(\alpha, \beta)$ уравнением $\alpha=\alpha(\beta)$, где $\beta \in[0,2 \pi]$ ( $\beta$ назовем „угловой“6 координатой $)$. Тогда вспомогательный контур определяется соотношениями $[13,14]$

$$
\alpha_{\Sigma}=\operatorname{Re} \eta, \quad \beta_{\Sigma}=\operatorname{Im} \eta, \quad \eta=\alpha+i \beta,
$$


где

$$
\beta=t+i \delta, \quad t \in[0,2 \pi] .
$$

В формуле $(8)\left(\alpha_{\Sigma}, \beta_{\Sigma}\right)-$ координаты „образа“ точки, расположенной на исходном контуре $S$, имеющей координаты $(\alpha, \beta), \delta$ - положительный параметр, отвечающий за степень деформации вспомогательного контура поперечного сечения тела. Для получения декартовых координат точки на вспомогательном контуре нужно использовать формулы

$$
x_{\Sigma}=\operatorname{Re} \xi, \quad y_{\Sigma}=\operatorname{Im} \xi,
$$

где, например, для полярных координат

$$
\xi(\eta)=\exp \eta=\exp (\alpha+i \beta), \quad(\alpha=\ln r, \quad \varphi=\beta),
$$

а для эллиптических координат (которые определяются по формулам $x=f \operatorname{ch} \alpha \cos \beta, y=f \operatorname{sh} \alpha \sin \beta)$

$$
\xi(\eta)=f \operatorname{ch} \eta=f \operatorname{ch}(\alpha+i \beta) .
$$

Заметим, что если $\delta=0$, то переменная $\xi \in C$, где $C-$ контур на комплексной плоскости $\xi$, соответствующий контуру сечения рассеивателя и конгруэнтный ему. Если начать увеличивать $\delta$, то $C$ будет сжиматься, и мы получим новый контур, который может быть выбран в качестве носителя вспомогательных источников $\Sigma$.

Далее приведем формулы для нахождения нормали к контуру тела, а именно, если обозначить через $n_{\alpha}, n_{\beta}-$ координаты нормали к контуру $\Sigma$, то

$$
n_{\alpha}=\frac{\dot{\beta}_{\Sigma}}{\sqrt{\dot{\alpha}_{\Sigma}^{2}+\dot{\beta}_{\Sigma}^{2}}}, \quad n_{\beta}=-\frac{\dot{\alpha}_{\Sigma}}{\sqrt{\dot{\alpha}_{\Sigma}^{2}+\dot{\beta}_{\Sigma}^{2}}} .
$$

Здесь через $\dot{\alpha}_{\Sigma}$ и $\dot{\beta}_{\Sigma}$ обозначены следующие производные:

$$
\begin{gathered}
\dot{\alpha}_{\Sigma}(t)=\operatorname{Re}(\dot{\alpha}(t+i \delta)+i), \\
\dot{\beta}_{\Sigma}=\operatorname{Im}(\dot{\alpha}(t+i \delta)+i), \quad t \in[0,2 \pi],
\end{gathered}
$$

где точка в правых частях равенств (14) означает производную функции $\dot{\alpha}=\alpha(t+i \delta)$ по аргументу $\beta=t+i \delta$, а в левых частях точка означает производную по параметру $t$. Заметим, что координаты нормали к исходному контуру тела получаются из формул (14), в которых нужно положить $\delta=0$.

Далее рассмотрим формулы для нахождения нормальной производной. Имеем

$$
\frac{\partial}{\partial n}=\frac{1}{h}\left(n_{\alpha} \frac{\partial}{\partial \alpha}+n_{\beta} \frac{\partial}{\partial \beta}\right),
$$

где $h=h_{\alpha}=h_{\beta}$ - коэффициенты Ламе выбранной системы координат. При этом

$$
\begin{aligned}
& \frac{1}{h} \frac{\partial}{\partial \alpha}=w_{11} \frac{\partial}{\partial x}+w_{12} \frac{\partial}{\partial y} \\
& \frac{1}{h} \frac{\partial}{\partial \beta}=w_{21} \frac{\partial}{\partial x}+w_{22} \frac{\partial}{\partial y}
\end{aligned}
$$

причем $w_{i j}$ - элементы матрицы

$$
\mathbf{T}=\left(\begin{array}{cc}
\frac{1}{\left|\xi^{\prime}\right|} \operatorname{Re} \xi^{\prime} & \frac{1}{\left|\xi^{\prime}\right|} \operatorname{Im} \xi^{\prime} \\
-\frac{1}{\left|\xi^{\prime}\right|} \operatorname{Im} \xi^{\prime} & \frac{1}{\left|\xi^{\prime}\right|} \operatorname{Re} \xi^{\prime}
\end{array}\right),
$$

где штрих означает производную от $\xi$ по переменой $\eta=\alpha+i \beta$. Таким образом, имеется единообразный подход (см. формулы $(8)-(17))$ для решения задачи дифракции на теле, контур поперечного сечения которого задан в подходящей ортогональной системе координат. Как показали результаты работ [13,14], при использовании подходящих координат удается добиться существенной (на несколько порядков большей) точности, чем, например, при решении задачи дифракции только в полярных координатах.

Основной трудностью при использовании ММДИ является выбор максимальной степени деформации вспомогательного контура, т.е. величины $\delta_{\max }$. Как следует из работ $[13,14]$, максимальное значение параметра деформации вспомогательного контура соответствует появлению у него точек самопересечения. Заметим, что приведенный ниже алгоритм является модификацией метода, рассмотренного в работе [14]. Для определения максимальной степени деформации, необходимой для построения вспомогательного контура $\Sigma$, используется алгоритм, аналогичный методу половинного деления для решения нелинейных уравнений. Сначала зададим некоторое положительное значение $\delta_{0}$, при котором вспомогательный контур имеет самопересечения. Для проверки наличия точек самопересечения вспомогательного контура мы решали уравнение

$$
F\left(t_{1}, t_{2}\right)=0
$$

где

$$
F\left(t_{1}, t_{2}\right)=\left\{\begin{array}{l}
\frac{\xi\left(t_{2}+i \delta\right)-\xi\left(t_{1}+i \delta\right)}{t_{2}-t_{1}}, \quad t_{1} \neq t_{2}, \\
\xi^{\prime}\left(t_{1}+i \delta\right), \quad t_{1}=t_{2},
\end{array}\right.
$$

которое эквивалентно системе двух нелинейных уравнений

$$
\left\{\begin{array}{l}
\operatorname{Re} F\left(t_{1}, t_{2}\right)=0 \\
\operatorname{Im} F\left(t_{1}, t_{2}\right)=0
\end{array}\right.
$$

При наличии действительных решений данной системы контур имеет точки самопересечения (очевидно, при этом $t_{1} \neq t_{2}$ ). Как показывают численные исследования, при увеличении параметра $\delta$ точки самопересечения контура $\Sigma$ будут появляться вблизи максимумов или минимумов исходного контура $S$ сечения тела. Поэтому для нахождения начальных приближений системы (19) можно поступить следующим образом. Вначале находим значения параметра $t_{0}$, при которых функция $\alpha=\alpha(t)$ имеет локальные экстремумы. Далее задаем начальные приближения к корням системы (19) в виде $t_{0} \pm \Delta$. Здесь величина $\Delta$ подбирается при помощи численных экспериментов. 
После нахождения величины $\delta_{0}$ применяем алгоритм, приведенный в [14]. А именно рассмотрим интервал $\left[0, \delta_{0}\right]$ изменения параметра $\delta$. Разделим этот интервал пополам и проверим, имеет ли уравнение (18) действительные корни для значения $\delta$ в середине исходного интервала. Если это условие выполнено, то берем левую половину интервала, если нет, то берем правую половину, и снова делим ее пополам, и так далее. Алгоритм останавливается, когда длина интервалов, на которые делится исходный интервал $\left[0, \delta_{0}\right]$, становится меньше заданного малого значения. В итоге за $\delta_{\max }$ берем левую границу интервала, рассмотренного на последней итерации.

В случае, если неровности границы представляют собой случайную функцию координат, можно рассмотреть усредненную диаграмму рассеяния волнового поля. Ниже приведены численные результаты усредненной диаграммы для случая дифракции на теле с сечением в виде „возмущенного“ многолистника

$$
r(\varphi)=\alpha(1+\tau \cos (p \varphi)+\varepsilon \cos (q \varphi+\psi)),
$$

где предполагается, что $|\varepsilon| \ll|\tau|<1$, а также эллиптического многолистника, задаваемого уравнением

$$
\alpha(\beta)=\alpha_{0}(1+\tau \cos (p \beta)+\varepsilon \cos (q \beta+\psi))
$$

в эллиптических координатах. Здесь $\varepsilon$ и $\psi-$ независимые случайные величины, а остальные величины неслучайны. Заметим, что при $\varepsilon=\tau=0$ многолистник (21) переходит в эллипс. В формуле (21) обозначено $\alpha_{0}=\ln \left(\frac{a+b}{f}\right)$, где $a, b-$ полуоси „невозмущенного“ эллипса и его межфокусное расстояние $f=\sqrt{b^{2}-a^{2}}$. Будем считать, что фаза $\psi$ в формулах (20) и (21) распределена равномерно в интервале $[0,2 \pi]$, а амплитуда возмущений $\varepsilon$ является случайной величиной, распределенной по усеченному нормальному закону, который имеет вид [15]

$$
w(\varepsilon)=\left\{\begin{array}{l}
C_{0} \exp \left(-\varepsilon^{2} / 2 \sigma_{0}^{2}\right), \quad|\varepsilon| \leq \varepsilon_{\max }, \\
0, \text { else, }
\end{array}\right.
$$

где величина $C_{0}$ определяется из условия нормировки. В дальнейшем будем считать, что $\varepsilon_{\max } \gg \sigma_{0}$. В результате закон распределения (22) будет близок к нормальному закону.

Усредненная диаграмма рассеяния вычисляется по формуле

$$
\langle g(\varphi)\rangle=\frac{1}{2 \pi} \int_{0}^{2 \pi} \int_{-\varepsilon_{\max }}^{\varepsilon_{\max }} w(\varepsilon) g(\varphi, \varepsilon, \psi) d \varepsilon d \psi,
$$

где $g(\varphi, \varepsilon, \psi)$ - диаграмма рассеяния, определяемая по формуле

$$
\begin{aligned}
g(\varphi, \varepsilon, \psi)= & -\frac{i}{4} \sum_{n=1}^{N} c_{n}(\varepsilon, \psi) \exp \left(i k \left(x_{\Sigma}\left(\beta_{n}\right) \cos \varphi\right.\right. \\
& \left.\left.+y_{\Sigma}\left(\beta_{n}\right) \sin \varphi\right)\right) .
\end{aligned}
$$

Здесь $c_{n}(\varepsilon, \psi)$ - амплитуды дискретных источников. В формуле (23) мы подчеркнули зависимость диаграммы от величин $\varepsilon$ и $\psi$. Для нахождения интегралов (23) можно использовать квадратурные формулы Гаусса и Эрмита (в последнем случае мы используем близость закона распределения (22) к нормальному закону).

\section{Локализация особенностей продолжения волнового поля на примере решения задачи дифракции на телах с сечением в виде многолистников}

При рассмотрении различных задач дифракции на телах, граница которых описывается вещественной аналитической функцией, возникает вопрос об аналитическом продолжении волновых полей в область, занимаемую телом. Как указано выше, аналитическое продолжение рассеянного поля можно выполнить при помощи аналитической деформации границы тела. При этом ключевую роль играют особенности продолжения волнового поля, расположенные внутри контура сечения тела (при решении внешних задач). В работе [9] показано, что если контур сечения тела задан в полярной системе координат, то особенности продолжения волнового поля можно найти как решения следующего уравнения:

$$
\xi^{\prime}(\varphi) \equiv\left(r^{\prime}(\varphi)+i r(\varphi)\right) \exp (i \varphi)=0,
$$

которое эквивалентно совокупности уравнений

$$
r^{\prime}(\varphi)+i r(\varphi)=0, \quad \exp (i \varphi)=0 .
$$

В частности, если сечение тела имеет вид многолистника (20), у которого будем предполагать фазу $\psi$ равной нулю, уравнение особенностей имеет вид

$$
\begin{aligned}
1 & +\frac{\tau}{2}(1+p) \gamma^{p}+\frac{\tau}{2}(1-p) \gamma^{-p} \\
& +\frac{\varepsilon}{2}(1+q) \gamma^{q}+\frac{\varepsilon}{2}(1-q) \gamma^{-q}=0,
\end{aligned}
$$

где $\gamma=\exp (i \varphi)$. В случае, когда $\varepsilon=0$, решения уравнения (27) приведены в книге [9]. В общем случае уравнение (27) решается численно.

Далее рассмотрим, например, особенности для так называемого чебышевского многолистника [16], заданного в эллиптических координатах уравнением

$$
\alpha(\beta)=\ln \left(s_{0}\left(1+\tau_{1} \cos (p \beta)+\varepsilon_{1} \cos (q \beta)\right)\right),
$$

где параметр $s_{0}=\exp \left(\alpha_{0}\right)$. Заметим, что при $\left|\tau_{1}\right| \ll 1$, $\left|\varepsilon_{1}\right| \ll 1$ уравнение (28) переходит в формулу (21), в которой $\tau_{1}=\alpha_{0} \tau, \varepsilon_{1}=\alpha_{0} \varepsilon, \psi=0$. Уравнение особенностей для многолистника (28) имеет вид (см. формулу (12)) [14]

$$
\xi^{\prime}(\beta) \equiv f \operatorname{sh}(\alpha+i \beta)\left(\alpha^{\prime}(\beta)+i\right)=0 .
$$


Уравнение (29) эквивалентно совокупности уравнений

$$
\alpha^{\prime}(\beta)+i=0, \quad \operatorname{sh}(\alpha+i \beta)=0 .
$$

Второе уравнение в системе (30) определяет особенности на комплексной плоскости $\xi$, имеющие координаты $( \pm f, 0)$. Эти особенности расположены в фокуcax „невозмущенного“ эллипса, в который переходит многолистник (28) при $\tau_{1}=\varepsilon_{1}=0$. Первое уравнение в (30) вновь преобразуется к виду (27), но в этом случае $\gamma=\exp (i \beta)$. Таким образом, имеется удобный алгоритм нахождения особенностей продолжения волнового поля для многолистников различного вида.

\section{Решение задачи дифракции на шероховатом круговом цилиндре при помощи Мду}

Рассмотрим частный случай рассмотренной выше задачи, а именно дифракцию плоской волны на шероховатом цилиндрическом теле с круговым сечением со средним радиусом $a$. Будем считать, что уравнение границы сечения цилиндра имеет вид

$$
r(\varphi)=a+\xi(\varphi),
$$

где $\xi(\varphi)$ - малая по модулю центрированная случайная функция, имеющая заданную корреляционную функцию

$$
\left\langle\xi(\varphi) \xi\left(\varphi^{\prime}\right)\right\rangle \equiv \sigma^{2} K\left(\varphi-\varphi^{\prime}\right),
$$

причем $\sigma^{2}=\left\langle\xi^{2}\right\rangle$ и $k \sigma \ll 1$. Таким образом, мы предполагаем, что корреляционная функция возмущений границы тела зависит только от аргумента $\varphi-\varphi^{\prime}$ [1]. Будем вновь рассматривать случай, когда на границе тела выполнено импедансное условие (2). Решим задачу при помощи метода малых возмущений на основе МДУ $[9,11,17]$. Заметим, что описанная ниже методика может быть распространена на задачу рассеяния на теле достаточно произвольной геометрии, если невозмущенная граница тела является слабо невыпуклой [9].

Представим полное поле вне тела в виде

$$
U=\langle U\rangle+\hat{U},
$$

где $\langle U\rangle-$ среднее поле и $\hat{U}-$ флуктуационное поле. Тогда, как показано в [1], имеют место следующие граничные условия при $r=a$ :

$$
\left\{\begin{array}{l}
\frac{\partial\langle U\rangle}{\partial r}=i k Z\langle U\rangle+\frac{1}{a^{2}}\left\langle\xi^{\prime} \frac{\partial U}{\partial \varphi}\right\rangle-\left\langle\xi \frac{\partial^{2} U}{\partial r^{2}}\right\rangle, \\
\frac{\partial \tilde{U}}{\partial r}=\frac{1}{a^{2}} \xi^{\prime} \frac{\partial\langle U\rangle}{\partial \varphi}-\xi \frac{\partial^{2}\langle U\rangle}{\partial r^{2}},
\end{array}\right.
$$

где штрих означает производную по $\varphi$. Диаграмма рассеяния тела имеет вид

$$
\begin{aligned}
g(\varphi) \equiv & \langle g(\varphi)\rangle+\tilde{g}(\varphi)=\sum_{n=-\infty}^{\infty}\left\langle a_{n}\right\rangle \exp (\operatorname{in} \varphi) \\
& +\sum_{n=-\infty}^{\infty} \tilde{a}_{n} \exp (\operatorname{in} \varphi),
\end{aligned}
$$

где $\tilde{\alpha}_{n}-$ случайные величины. При этом, как следует из результатов работ $[9,10]$, справедливы формулы

$$
\begin{aligned}
\left\langle a_{n}\right\rangle & =\left.\frac{i a}{4} \int_{0}^{2 \pi}\left[\frac{\partial\langle U\rangle}{\partial r}-\langle U\rangle \frac{\partial}{\partial r}\right]\right|_{r=a} \\
& \times J_{m}(k r) \exp \left(-i m\left(\varphi-\frac{\pi}{2}\right)\right) d \varphi, \\
\tilde{a}_{m} & =\left.\frac{i a}{4} \int_{0}^{2 \pi}\left[\frac{\partial \tilde{U}}{\partial r}-\tilde{U} \frac{\partial}{\partial r}\right]\right|_{r=a} \\
& \times J_{m}(k r) \exp \left(-i m\left(\varphi-\frac{\pi}{2}\right)\right) d \varphi .
\end{aligned}
$$

Подставим в формулы (34) производные $\frac{\partial\langle U\rangle}{\partial r}$ и $\frac{\partial \tilde{U}}{\partial r}$, которые получаются из граничных условий (32), если считать все остальные величины известными. Тогда получим

$$
\begin{aligned}
& \left\langle a_{m}\right\rangle=\left.\frac{i a}{4} \int_{0}^{2 \pi}\left[\frac{1}{a^{2}}\left\langle\xi^{\prime} \frac{\partial \tilde{U}}{\partial \varphi}\right\rangle-\left\langle\xi \frac{\partial^{2} \tilde{U}}{\partial r^{2}}\right\rangle\right]\right|_{r=a} \\
& \times J_{m}(k a) \exp \left(-i m\left(\varphi-\frac{\pi}{2}\right)\right) d \varphi \\
& -\frac{i a}{4} \int_{0}^{2 \pi}\left[J_{m}^{\prime}(k a)-\left.i k Z J_{m}(k a)\langle U\rangle\right|_{r=a} \exp \left(-i m\left(\varphi-\frac{\pi}{2}\right)\right) d \varphi\right. \\
& \left.\tilde{a}_{m}=\frac{i a}{4} \int_{0}^{2 \pi}\left[\frac{1}{a^{2}} \xi^{\prime} \frac{\partial\langle U\rangle}{\partial \varphi}\right\rangle-\xi \frac{\partial^{2}\langle U\rangle}{\partial r^{2}}\right]\left.\right|_{r=a} \\
& \times J_{m}(k a) \exp \left(-i m\left(\varphi-\frac{\pi}{2}\right)\right) d \varphi \\
& -\frac{i k a}{4} \int_{0}^{2 \pi}\left[J_{m}^{\prime}(k a)-\left.i k Z J_{m}(k a) \tilde{U}\right|_{r=a}\right. \\
& \times J^{\prime}(k a) \exp \left(-i m\left(\varphi-\frac{\pi}{2}\right)\right) d \varphi .
\end{aligned}
$$

Для дальнейшего решения задачи используем представление полей $\langle U\rangle$ и $\tilde{U}$ в виде рядов Рэлея:

$$
\begin{gathered}
\langle U\rangle=U^{0}+\sum_{n=-\infty}^{\infty} i^{-n}\left\langle a_{n}\right\rangle H_{n}^{(2)}(k a) \exp (i n \varphi), \\
\tilde{U}=\sum_{n=-\infty}^{\infty} i^{-n} \tilde{a}_{n} H_{n}^{(2)}(k a) \exp (\operatorname{in} \varphi) .
\end{gathered}
$$

Подставим разложения (38) в формулы (36) и (37) и спроектируем полученные равенства на базис Фурье. 
Тогда получим следующие системы линейных алгебраических уравнений (СЛАУ) относительно коэффициентов $\left\langle a_{n}\right\rangle$ и $\tilde{a}_{n}$ :

$$
\begin{gathered}
\left\langle a_{m}\right\rangle=a_{m}^{0}+\sum_{n=-\infty}^{\infty} G_{m n}^{\mathrm{I}}\left\langle a_{n}\right\rangle+\sum_{n=-\infty}^{\infty}\left\langle\tilde{G}_{m n} \tilde{a}_{n}\right\rangle, \\
m=0, \pm 1, \pm 2, \ldots \\
\tilde{a}_{m}=\tilde{a}_{m}^{0}+\sum_{n=-\infty}^{\infty} G_{m n}^{\mathrm{II}} \tilde{a}_{n}+\sum_{n=-\infty}^{\infty} \tilde{G}_{m n}\left\langle a_{n}\right\rangle \\
m=0, \pm 1, \pm 2, \ldots
\end{gathered}
$$

Здесь

$$
\begin{gathered}
G_{m n}^{\mathrm{I}}=-\frac{i \pi k a}{2} H_{n}^{(2)}(k a)\left(J_{m}^{\prime}(k a)-i k Z J_{m}(k a)\right) \delta_{m n} \\
G_{m n}^{\mathrm{II}}=-\frac{i \pi k a}{2} J_{m}^{\prime}(k a) H_{n}^{(2)}(k a) \delta_{m n} \\
\tilde{G}_{m n}=\frac{i \pi}{2 a} i^{m-n}\left(n(n-m) H_{n}^{(2)}(k a)\right. \\
\left.-(k a)^{2} H_{n}^{(2)^{\prime \prime}}(k a)\right) J_{m}(k a) \xi_{m-n} \\
a_{m}^{0}=-\frac{i \pi k a}{2} i^{m} J_{m}(k a)\left(J_{m}^{\prime}(k a)-i k Z J_{m}(k a)\right) \exp \left(-i m \varphi_{0}\right), \\
\tilde{a}_{m}^{0}=\frac{i \pi}{2 a} \sum_{l=-\infty}^{\infty} i^{m-l}\left(l(l-m) J_{l}(k a)\right. \\
\left.-(k a)^{2} J_{l}^{\prime \prime}(k a)\right) J_{m}(k a) \exp \left(-i m \varphi_{0}\right) \xi_{m-l} \\
\xi_{n}=\frac{1}{2 \pi} \int_{0}^{2 \pi} \zeta(\varphi) \exp (-i n \varphi) d \varphi
\end{gathered}
$$

В формулах (41) и (42) $\delta_{m n}-$ символ Кронекера. Пользуясь тем, что матрица $\left\|G_{m n}^{\mathrm{II}}\right\|$ не случайна и является диагональной, выразим величины $\tilde{a}_{n}$ из формулы (40) и подставим их в (39). В силу того, что функция $K$ зависит только от $\varphi-\varphi^{\prime}$, нетрудно показать, что будет справедлива следующая формула для усредненной диаграммы рассеяния:

$$
\langle g(\varphi)\rangle=\sum_{n=-\infty}^{\infty} \frac{b_{n}^{0}}{1-Q_{n}} \exp (\operatorname{in} \varphi),
$$

где

$$
\begin{gathered}
Q_{n}=\sigma^{2} \sum_{l=-\infty}^{\infty} g_{n l}^{\mathrm{I}} g_{l n}^{\mathrm{II}} K_{n-l}, \\
b_{n}^{0}=a_{n}^{\infty}+\sum_{l=-\infty}^{\infty} g_{n l}^{\mathrm{I}} g_{n l}^{0} K_{n-l} .
\end{gathered}
$$

В формулах (48) и (49) обозначено

$$
g_{n l}^{\mathrm{I}}=\frac{i^{n-l}\left(l(l-n) H_{l}^{(2)}(k a)-(k a)^{2} H_{l}^{(2)^{\prime \prime}}(k a)\right)}{k a^{2}\left(H_{n}^{(2))^{\prime}}(k a)-i k Z H_{n}^{(2)}(k a)\right)},
$$

$$
\begin{gathered}
g_{n l}^{\mathrm{II}}=\frac{i^{l-n}\left(n(n-l) H_{n}^{(2)}(k a)-(k a)^{2} H_{n}^{(2)^{\prime \prime}}(k a)\right)}{k a^{2}\left(H_{l}^{(2)^{\prime}}(k a)\right.} \\
g_{n l}^{0}=\frac{i^{l-n}\left(n(n-l) J_{n}(k a)-(k a)^{2} J_{n}^{\prime \prime}(k a)\right) \exp \left(-i n \varphi_{0}\right)}{k a^{2}\left(H_{l}^{(2)^{\prime}}(k a)\right.} \\
a_{n}^{\infty}=-i^{n} \frac{J_{n}^{\prime}(k a)-i k Z J_{n}(k a)}{H_{n}^{(2)^{\prime}}(k a)-i k Z H_{n}^{(2)}(k a)} \exp \left(-i n \varphi_{0}\right) \\
K_{n-l}=\frac{1}{2 \pi} \int_{0}^{2 \pi} K(t) \exp (-i(n-l) t) d t
\end{gathered}
$$

Таким образом, формулы (47)-(54) дают явное решение задачи дифракции на шероховатом круговом цилиндре в рамках ММВ. Отметим, что величины $a_{n}^{\infty}$ в формуле (49) представляют собой коэффициенты Фурье невозмущенной диаграммы (которая получается при $\sigma=0)$, а остальные величины обусловлены возмущениями границы тела.

\section{Результаты вычислений}

Для тестирования методик расчета усредненной диаграммы рассеяния на основе ММДИ и МДУ была рассчитана угловая зависимость модуля усредненной диаграммы рассеяния для задачи дифракции плоской волны на шероховатом круговом цилиндре. Случайные возмущения границы тела моделировались функцией

$$
r(\varphi)=a(1+\varepsilon \cos (6 \varphi+\psi)),
$$

где амплитуда $\varepsilon$ и фаза $\psi$ распределены так, как описано выше. В этом случае корреляционная функция возмущений границы имеет вид

$$
\sigma^{2} K\left(\varphi-\varphi^{\prime}\right)=\frac{1}{2} a^{2} \sigma_{0}^{2} \cos \left(6\left(\varphi-\varphi^{\prime}\right)\right) .
$$

Заметим, что в силу близости распределения (22) к нормальному закону можно считать, что $\left\langle\varepsilon^{2}\right\rangle \approx \sigma_{0}^{2}$.

На рис. $1, a$ и $1, b$ представлены угловые зависимости модуля усредненной диаграммы рассеяния шероховатого кругового цилиндра для случаев Е- и Н-поляризации падающего поля соответственно. Сплошной кривой на рисунках изображена зависимость усредненной диаграммы, полученной при помощи ММДИ, точками показаны зависимости диаграммы, полученной при помощи ММВ на основе МДУ, а штриховой кривой - зависимости диаграммы для невозмущенного кругового цилиндра (при $\sigma=0)$. Радиус кругового цилиндра был равен $k a=5$. Угол падения плоской волны $\varphi_{0}=45^{\circ}$. Величина среднеквадратичного отклонения амплитуды неровностей границы составляла $\sigma_{0}=0.1$ в случае решения задачи дифракции Е-поляризованной волны и $\sigma_{0}=0.05$ в случае Н-поляризации. Таким образом, величина среднеквадратичного отклонения функции $\xi(\varphi)$, т.е. $k \sigma$, равнялась приблизительно 0.35 и 0.177 для рис. $1, a$ и 

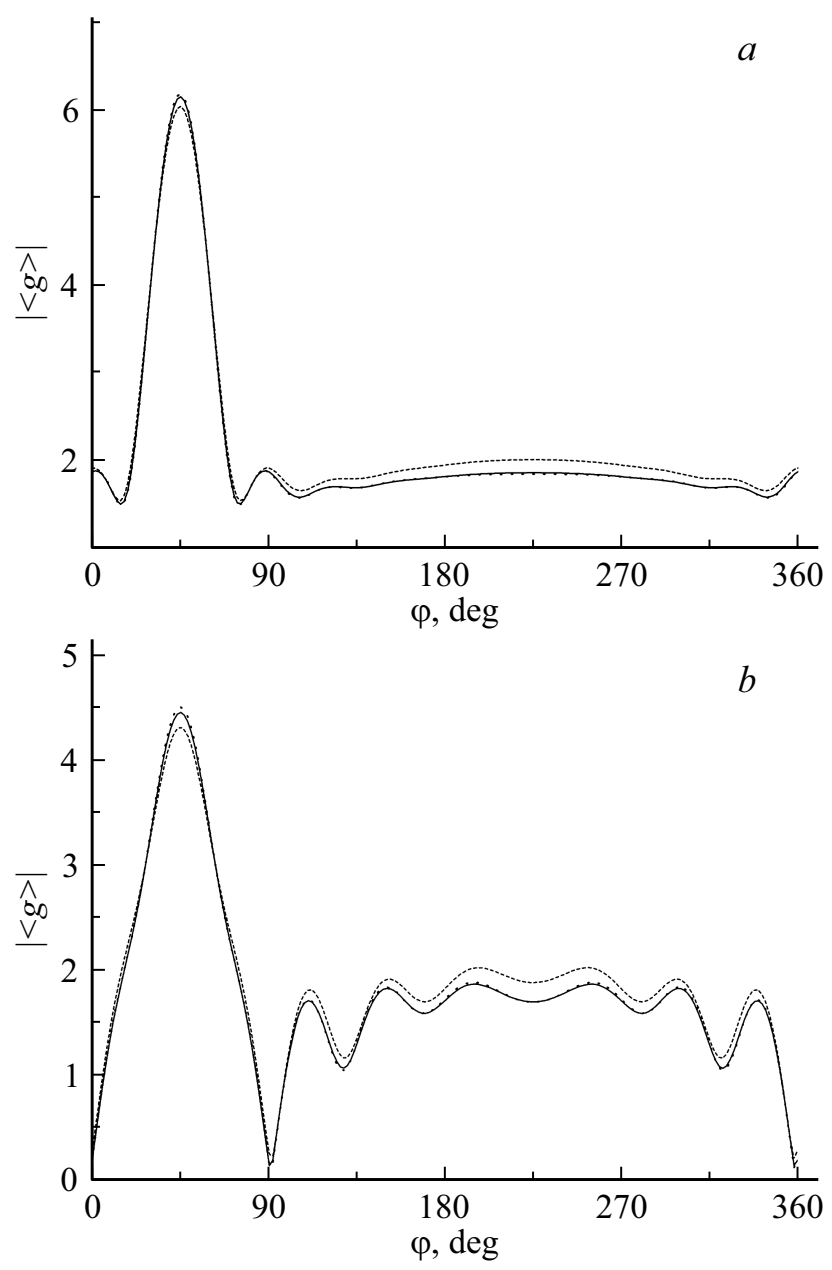

Рис. 1. Угловые зависимости модуля усредненной диаграммы рассеяния для задачи дифракции на шероховатом круговом цилиндре для случая Е-поляризации $(a)$ и Н-поляризации $(b)$ падающего поля.

$1, b$ соответственно. Как видно из приведенных рисунков, результаты расчета усредненной диаграммы, полученные на основе строгого подхода и при помощи ММВ, отличаются незначительно. В то же время диаграмма невозмущенного тела достаточно сильно отличается от усредненной диаграммы в направлении обратного рассеяния. Таким образом, методика на основе приближенного подхода дает удовлетворительные результаты при достаточно больших значениях величины $k \sigma$.

Далее рассмотрим результаты усреднения диаграммы рассеяния, полученные при помощи ММДИ. Была решена задача дифракции на теле со случайной границей, описываемой уравнениями (20) и (21). На pис. $2, a$ и $2, b$ показаны реализации случайных контуров тела с сечением в виде многолистника, заданного в полярных и эллиптических координатах соответственно (сплошные кривые на рисунке). Размер сечения тела в виде многолистника, заданного в полярных координатах, $k a=10$, параметры $p=4, q=16, \tau=1 / 3$, $\varepsilon=\sigma_{0}=0.05, \psi=2$. В случае тела с сечением в виде
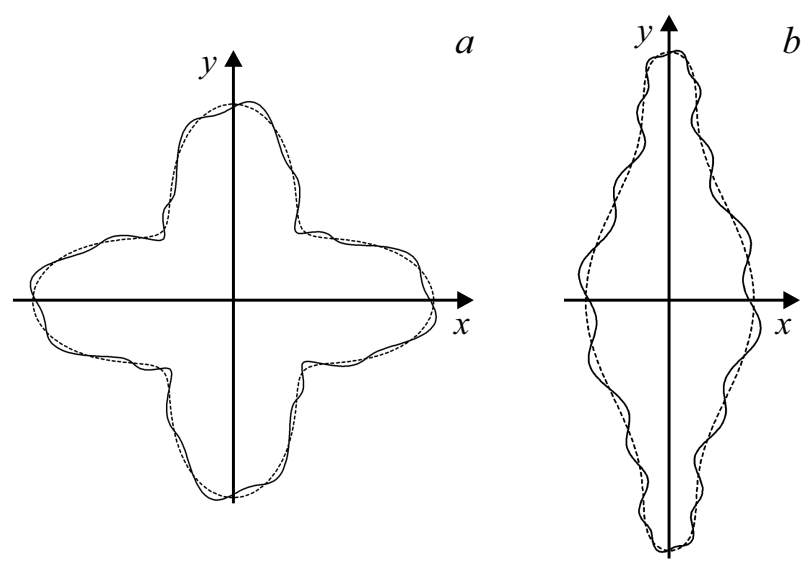

Рис. 2. Геометрия одной из реализаций контура сечения тела в виде многолистника, заданного в полярных $(a)$ и эллиптических $(b)$ координатах.

эллиптического многолистника параметры геометрии были следующими: $k a=3.25, k b=13, p=4, q=16$, $\tau=1 / 3, \varepsilon=\sigma_{0}=0.147, \quad \psi=2$. На этих же рисунках представлены геометрии „невозмущенного“ контура тела при $\varepsilon=0$ (штриховые кривые). Отметим, что величины $\sigma_{0}$ для указанных рассеивателей выбраны так, чтобы максимальные значения среднеквадратичного отклонения неровностей границы тел были примерно одинаковы для обоих типов многолистников. Размеры тел также согласованы таким образом, чтобы диаметры „невозмущенных“ рассеивателей были одинаковы.

Рисунки 3, 4 иллюстрируют угловые зависимости модуля усредненной диаграммы рассеяния для указанных многолистников для углов падения $\varphi_{0}=0$ (рис. $3, a$ и $4, a)$ и $\varphi_{0}=45^{\circ}$ (рис. $3, b$ и $4, b$ ). При этом рис. 3 относится к случаю дифракции на теле с сечением вида (20), а рис. 4 соответствует рассеянию на теле с сечением вида (21). Штриховыми кривыми на рисунках показаны угловые зависимости модуля диаграммы рассеяния гладкого тела. Рассматривался случай $\mathrm{H}$ поляризации. Как видно из рисунков, диаграммы шероховатого тела достаточно сильно отличаются от соответствующих диаграмм тела с „невозмущенной“ границей. При этом максимальное различие диаграмм достигается в окрестности направления обратного рассеяния. Как показывают расчеты, в случае Е-поляризации различие усредненной диаграммы и диаграммы „невозмущенного“ тела существенно меньше для выбранных параметров задачи. Отметим, что точность получаемых результатов контролировалась при помощи проверки оптической теоремы для нескольких реализаций контура тела, а также проверки выполнения краевого условия на контуре тела посередине между точками коллокации (т.е. вычисления невязки краевого условия). Расчеты показали, что невязка краевого условия не превосходила $5 \cdot 10^{-3}$, а оптическая теорема выполнялась с точностью не ниже $10^{-6}$. 

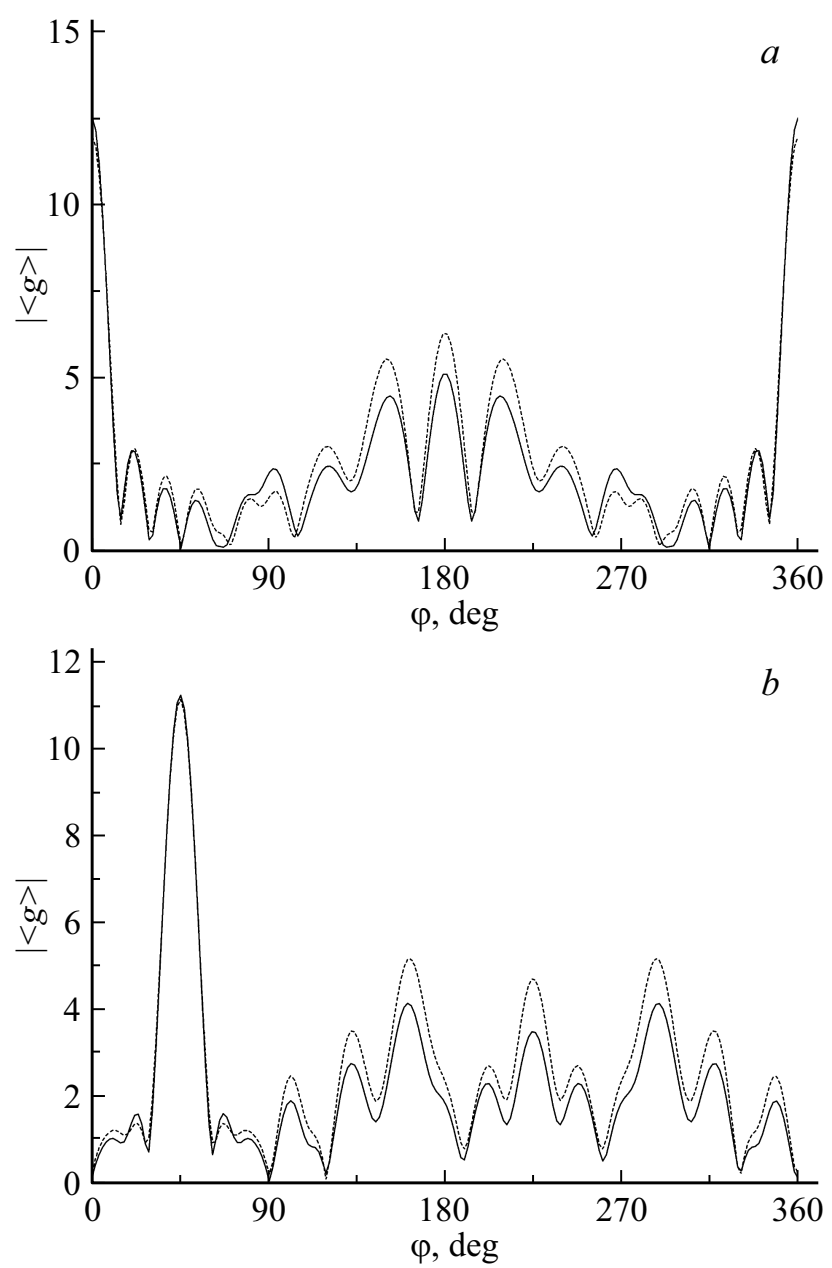

Рис. 3. Угловые зависимости модуля усредненной диаграммы рассеяния шероховатого тела с сечением вида (20) для угла падения $\varphi_{0}=0(a)$ и $\varphi_{0}=45^{\circ}(b)$.

Рассмотрим, наконец, результаты, касающиеся степени „зеркальности“ цилиндрического тела с сечением в виде указанных выше многолистников. На рис. 5 приведены геометрии контура сечения тела и вспомогательного контура, получаемого путем аналитической деформации исходного контура сечения тела, для случая многолистников вида (20) и (21). Рисунок 5,a соответствует значению $\tau=0.1$, а рис. $5, b-\tau=0.7$. Рисунок 5,c относится к случаю рассеяния на теле с сечением вида (21), причем $\tau=1 / 3$. Размеры сечения тел были следующие. Для многолистника вращения вида (20) $k a=12.12$ при $\tau=0.1$ и $k a=7.84$ при $\tau=0.7$, a размеры сечения тела вида (21) имели значения $k a=3.25, k b=13$. Параметры $p, q$ были такими же, как и выше. При этом фаза $\psi$ была равна нулю, а параметр $\varepsilon=0.0083$ для многолистника (20) в случае $\tau=0.1$ и $\varepsilon=0.0128$ для многолистника (20) в случае $\tau=0.7$ и $\varepsilon=0.029$ для многолистника вида (21) (т.е. рассматривались детерминированные возмущения границы тела). Амплитуды неровностей границы выбраны таким образом, что максимальная разность
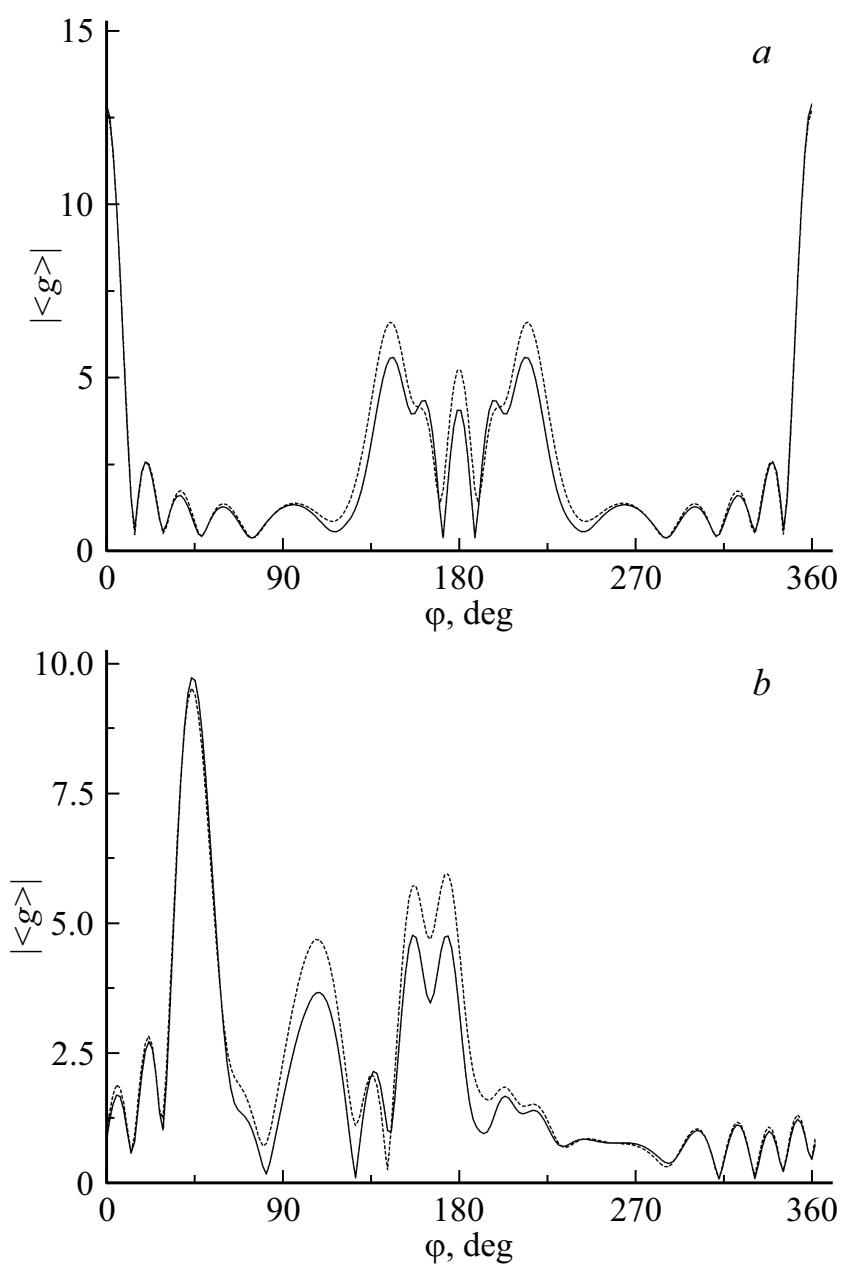

Рис. 4. Угловые зависимости модуля усредненной диаграммы рассеяния шероховатого тела с сечением вида (21) для угла падения $\varphi_{0}=0(a)$ и $\varphi_{0}=45^{\circ}(b)$.

координат точек контура шероховатого тела и точек контура „невозмущенного“ тела были одинаковы для обоих типов многолистников. Штриховыми кривыми показаны геометрии контура сечения и вспомогательного контура „невозмущенного“ (гладкого) тела. Заметим, что вспомогательные контуры, показанные на рисунках, соответствуют максимальному значению $\delta_{\max }$ параметра деформации контура сечения тела. Как указано в работе [14], особые точки продолжения волнового поля необходимо соединить разрезами, геометрия которых определяет условие самопересечения вспомогательного контура, а именно этот контур не должен пересекать данные разрезы. Таким образом, условие самопересечения вспомогательного контура тесно связано с геометрией множества особенностей продолжения волнового поля внутрь контура сечения тела [9]. Как видно из рис. 5, $a$, в случае тела с сечением вида (20) при $\tau=0.1$ вспомогательный контур шероховатого и „невозмущенного“ тела существенно различаются. В тоже время аналогичные контуры многолистника вида (20) при $\tau=0.7$ и эллиптического многолистника 

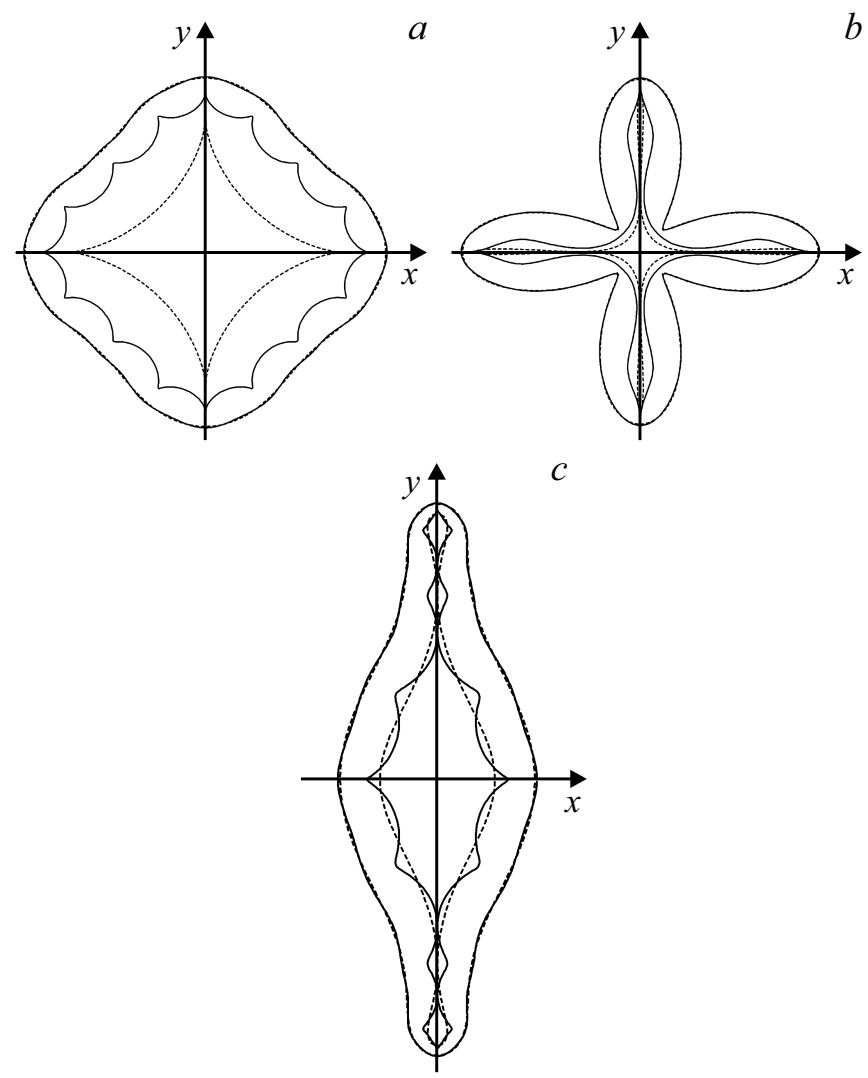

Рис. 5. Геометрия контура сечения и вспомогательного контура детерминированного шероховатого тела с сечением в виде многолистника, заданного в полярных $(a),(b)$ и эллиптических $(c)$ координатах.

близки друг к другу. Данное обстоятельство приводит к соответствующему поведению угловых зависимостей диаграмм рассеяния шероховатого и гладкого тел, которые изображены на рис. 6. Во всех трех случаях плоская волна падала вдоль оси $x$. Сплошные кривые на рисунке соответствуют дифракции на шероховатом теле, а штриховые кривые иллюстрируют зависимости диаграммы рассеяния для гладкого рассеивателя. Как следует из рисунков, диаграммы гладкого и шероховатого тел с сечением вида (20) при $\tau=0.1$ различаются в окрестности направления обратного рассеяния. В случае дифракции на теле с сечением вида (20) при $\tau=0.7$ и вида (21) соответствующие диаграммы близки друг к другу в большей части диапазона изменения угла наблюдения. Так как вспомогательный контур при $\delta=\delta_{\max }$,повисает“ на точках, являющихся особенностями аналитического продолжения поля внутрь контура тела, то можно сделать следующий вывод. В случае, если особенности продолжения волнового поля внутрь гладкого тела расположены достаточно близко к контуру сечения рассеивателя, то диаграммы рассеяния гладкого и шероховатого тел близки при условии малости неровностей границы по сравнению с длиной волны. Если же особенности продолжения поля расположены
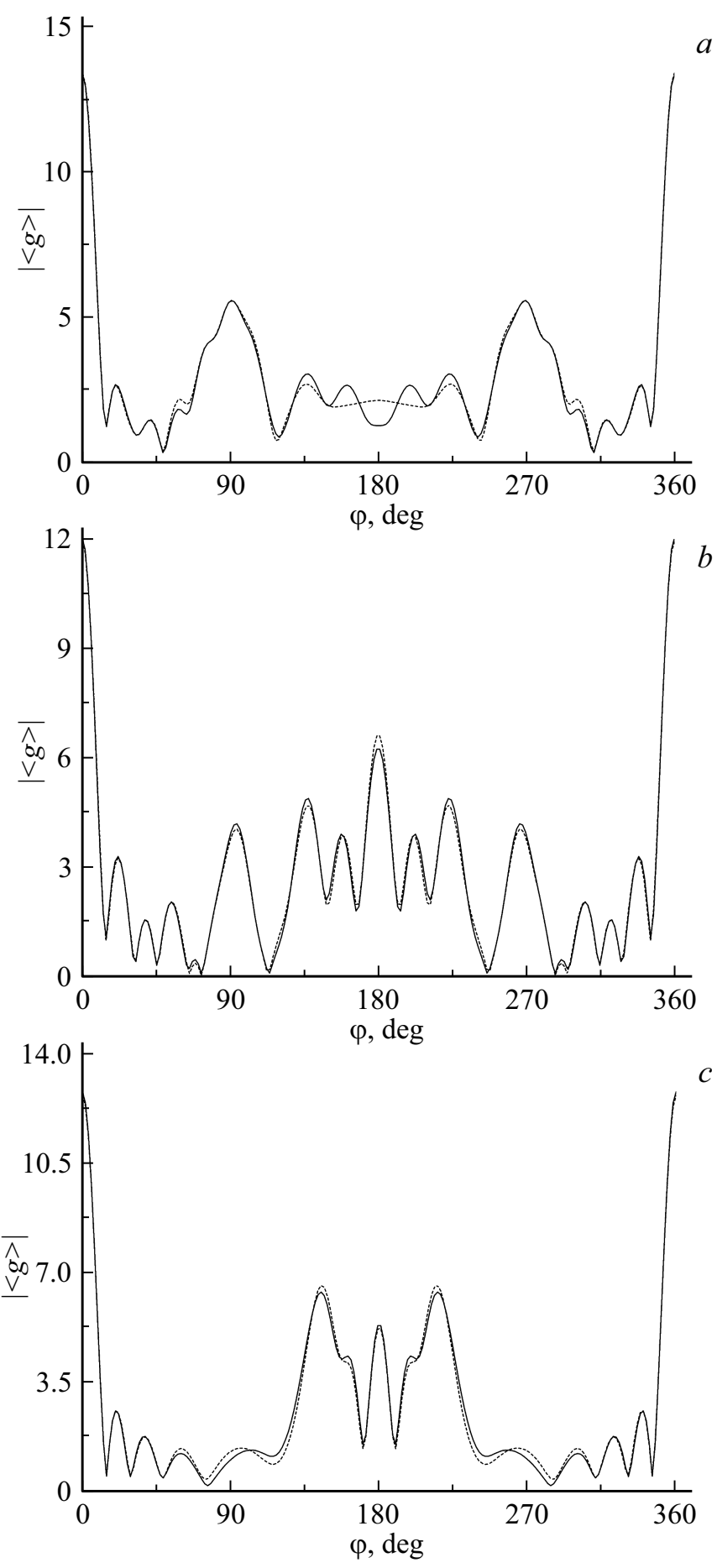

Рис. 6. Угловые зависимости модуля диаграммы рассеяния детерминированного шероховатого тела с сечением в виде многолистника, заданного в полярных $(a),(b)$ и эллиптических $(c)$ координатах.

„далеко“ от контура сечения гладкого тела, то диаграммы гладкого и шероховатого тел различаются достаточно сильно даже для малых амплитуд неровностей границы. 


\section{Заключение}

На основе ММДИ разработан численный алгоритм решения двумерной задачи дифракции на шероховатом цилиндрическом теле. Метод пригоден для расчета характеристик рассеяния тел с достаточно большими значениями амплитуды неровностей границы. В работе предложен эффективный алгоритм построения вспомогательного контура, являющегося носителем дискретных источников, применимый к широкому классу тел с аналитической границей.

В случае малых по сравнению с длиной волны неровностей границы приведен алгоритм, основанный на методе диаграммных уравнений. Рассмотрено уравнение для нахождения координат особенностей продолжения волнового поля внутрь рассеивателя на примере чебышевского многолистника.

Представлены угловые зависимости модуля усредненной диаграммы рассеяния для статистически неровного кругового цилиндра. Неровности границы моделировались синусоидальной случайной функцией, имеющей случайную амплитуду и фазу.

Показано хорошее совпадение результатов, полученных при помощи ММДИ и метода малых возмущений на основе МДУ.

Получены угловые зависимости усредненной диаграммы рассеяния для задачи дифракции на шероховатом теле с сечением в виде многолистника, заданного в полярных и эллиптических координатах. Численно исследовано влияние малых детерминированных неровностей границы на геометрию множества особенностей продолжения волнового поля внутрь контура сечения тела. Показано, во-первых, что геометрия множества указанных особенностей существенно зависит от формы тела (т.е. „невозмущенного“ контура сечения рассеивателя). Во-вторых, продемонстрировано, что при существенном различии множества особенностей гладкого и шероховатого тел диаграммы рассеяния также достаточно сильно различаются даже при малых значениях неровностей границы сечения тела.

\section{Финансирование работы}

Работа выполнена при поддержке Российского фонда фундаментальных исследований (проект № 19-0200654).

\section{Конфликт интересов}

Конфликт интересов отсутствует.

\section{Список литературы}

[1] Басс Ф.Г., Фукс И.М. Рассеяние волн на статистически неровной поверхности. М.: Наука, 1972. 424 с.

[2] Воронович А.Г. // Журн. эксп. теор. физ. 1985. Т. 89. С. 116.
[3] Воронович А.Г. // Акуст. журн. 2007. Т. 53. № 3. С. 346; Voronovich A.G. // Acoust. Phys. 2007. V. 53. N 3. P. 298.

[4] Muinonen K., Nousiainen T., Fast P., Lumme K., Peltoniemi J.I. // J. Quant. Spectrosc. Radiat. Transfer. 1996. V. 55. P. 577.

[5] Muinonen K. // Light Scattering by Nonspherical Particles / Ed. by Mishchenko M.I., Hovenier J.W., and Travis L.D. Academic Press, 2000. P. 323-354.

[6] Auger J.-C., Fernandes G., Aptowicz K., Pan Y.-L., Chang R. // Appl. Phys. 2010. V. 99. P. 229.

[7] Kahnert M., Rother T. // Opt Express. 2011. V. 19. P. 11138.

[8] Кюркчан А.Г., Минаев С.А., Соловейчик А.Л. // РЭ. 2001. T. 46. № 6. C. 666; Kyurkchan A.G., Minaev S.A. Soloveichik A.L. / J. Commun. Technol. Electron. 2001. V. 46. N 6. P. 615.

[9] Kyurkchan A.G., Smirnova N.I. Mathematical Modelling in Diffraction Theory Based on a Priori Information on the Analytical Properties of the Solution. Amsterdam: Elsevier, 2017.

[10] Маненков С.А. // Акуст. журнал. 2014. Т. 60. № 2. С. 129; Manenkov S.A. // Acoust. Phys. 2014. V. 60. N 2. P. 127.

[11] Кюркчан А.Г. // ДАН. 1992. Т. 325. № 2. С. 273; Kyurkchan A.G. // Soviet Physics Doklady. 1992. V. 37. N 7. P. 338.

[12] Кюркиан А.Г. // УФН. 2017. Т. 187. № 10. С. 1097; Kyurkchan A.G. // Physics - Uspekhi. 2017. V. 60. N 10. P. 1018.

[13] Kyurkchan A.G., Manenkov S.A. // J. Quant. Spectrosc. Radiat. Transfer. 2012. V. 113. P. 2368.

[14] Kyurkchan A.G., Manenkov S.A. // J. Quant. Spectrosc. Radiat. Transfer. 2018. V. 221. P. 243.

[15] Григорьев Г.И., Заборонкова Т.М., Коган Л.П. // РЭ. 2017. T. 50. № 5. C. 552; Grigor'ev G.I., Zaboronkova T.M., Kogan L.P. // J. Commun. Technol. Electron. 2017. V. 62. P. 1091.

[16] Фарафонов В.Г., Винокуров А.А., Барканов С.В. // Опт. и спектр. 2011. Т. 111. № 5. С. 852; Farafonov V.G., Vinokurov A.A., Barkanov S.V. // Opt. Spectrosc. 2011. V. 111. P. 824.

[17] Кюркчан А.Г., Маненков С.А. // РЭ. 2021. Т. 66. № 3. C. 226; Kyurkchan A.G., Manenkov S.A. // J. Commun. Technol. Electron. 2021. V. 66. N 3. P. 1091. 